\title{
The prospective GermanVasc cohort study
}

\section{Endovascular and open-surgical treatment of symptomatic peripheral artery disease}

\author{
Artur Kotov ${ }^{1}$, Frederik Peters ${ }^{1}$, Eike Sebastian Debus ${ }^{1}$, Thomas Zeller ${ }^{2}$, Peter Heider ${ }^{3}$, \\ Konstantinos Stavroulakis ${ }^{4}$, Jürgen Remig ${ }^{5}$, Andreas Gussmann ${ }^{6}$, Johannes Hoffmann ${ }^{7}$, \\ Oliver Friedrich ${ }^{8}$, Thomas Nolte ${ }^{9}$, and Christian-Alexander Behrendt ${ }^{1}$ (D) \\ ${ }^{1}$ University Medical Center Hamburg-Eppendorf, Hamburg, Germany \\ ${ }^{2}$ University Heart Center Freiburg-Bad Krozingen, Germany \\ ${ }^{3}$ Isar Clinic Munich, Germany \\ ${ }^{4}$ St. Franziskus Hospital GmbH, Münster, Germany \\ ${ }^{5}$ Bonn Community Hospital, Haus St. Petrus, Bonn, Germany \\ ${ }^{6}$ HELIOS Clinical Centre Berlin-Buch, Berlin, Germany \\ ${ }^{7}$ Contilia Elisabeth-Hospital Essen, Germany \\ ${ }^{8}$ GFO Clinics Bonn Operating St. Marien, Bonn, Germany \\ ${ }^{9}$ Bad Bevensen Heart and Vascular Centre, Bad Bevensen, Germany
}

\begin{abstract}
Summary: Background: Previous observational studies reported a wide variation and possible room for improvement in the treatment of patients suffering from symptomatic peripheral artery disease (PAD). Yet, systematic assessment of everyday clinical practice is lacking. A General Data Protection Regulation (GDPR) compliant registry was developed and used to collect comprehensive data on clinical treatment and outcomes regarding PAD in Germany. Here, we report baseline characteristics of patients prospectively enrolled until the end of 2020. Methods: The GermanVasc registry study is a prospective longitudinal multicentre cohort study. Between $1^{\text {st }}$ May 2018 and $31^{\text {st }}$ December 2020, invasive endovascular, open-surgical, and hybrid revascularisations of patients suffering from chronic symptomatic PAD were prospectively included after explicit informed consent (NCT03098290). For ensuring high quality of the data, we performed comprehensive risk-based and random-sample external and internal validation. Results: In total, 5608 patients from 31 study centres were included (34\% females, median 69 years). On-site monitoring visits were performed at least once in all centres. The proportion of chronic limb-threatening ischaemia was $30 \%$ and $13 \%$ were emergent admissions. 55\% exhibited a previous revascularisation. Endovascular techniques made $69 \%$ among all documented invasive procedures $(n=6449)$. Thirty-five percent were classified as patients with severe systemic disease, and $3 \%$ exhibited a constant threat to life according to the American Society of Anaesthesiologists classification. The risk profile comprised of $75 \%$ former or current smokers, $36 \%$ diabetes mellitus, and in 30\% a current ischemic heart disease was present. At discharge, $93 \%$ of the patients received antiplatelets and $77 \%$ received statins. Conclusions: The GermanVasc registry study provides insights into real-world practice of treatment and outcomes of 5,608 patients with symptomatic PAD in Germany. The cohort covers a broader range of disease severity and types of interventions than usually found in trials. In future studies, comparative outcomes will be analysed in more detail.
\end{abstract}

Keywords: Health services research, peripheral artery disease, intermittent claudication, chronic limb-threatening ischaemia, endovascular techniques, bypass surgery

\section{Introduction}

Although peripheral artery disease (PAD) is a common illness with more than 230 million affected worldwide, the existing evidence base to treat this target population is still incomplete in regard of patient selection, best medical treatment, and the best patient-centred approach to revascularise atherosclerotic lesions [1].
A wide variation between countries was previously reported concerning the proportion of patients with claudication, endovascular techniques, females, and octogenarians [2]. In line with this unexplained variation, nearly half of all recommendations in valid practice guidelines are based on low level of evidence $[3,4,5]$.

While the vascular community waits with bated breath for the first results of currently recruiting randomized 

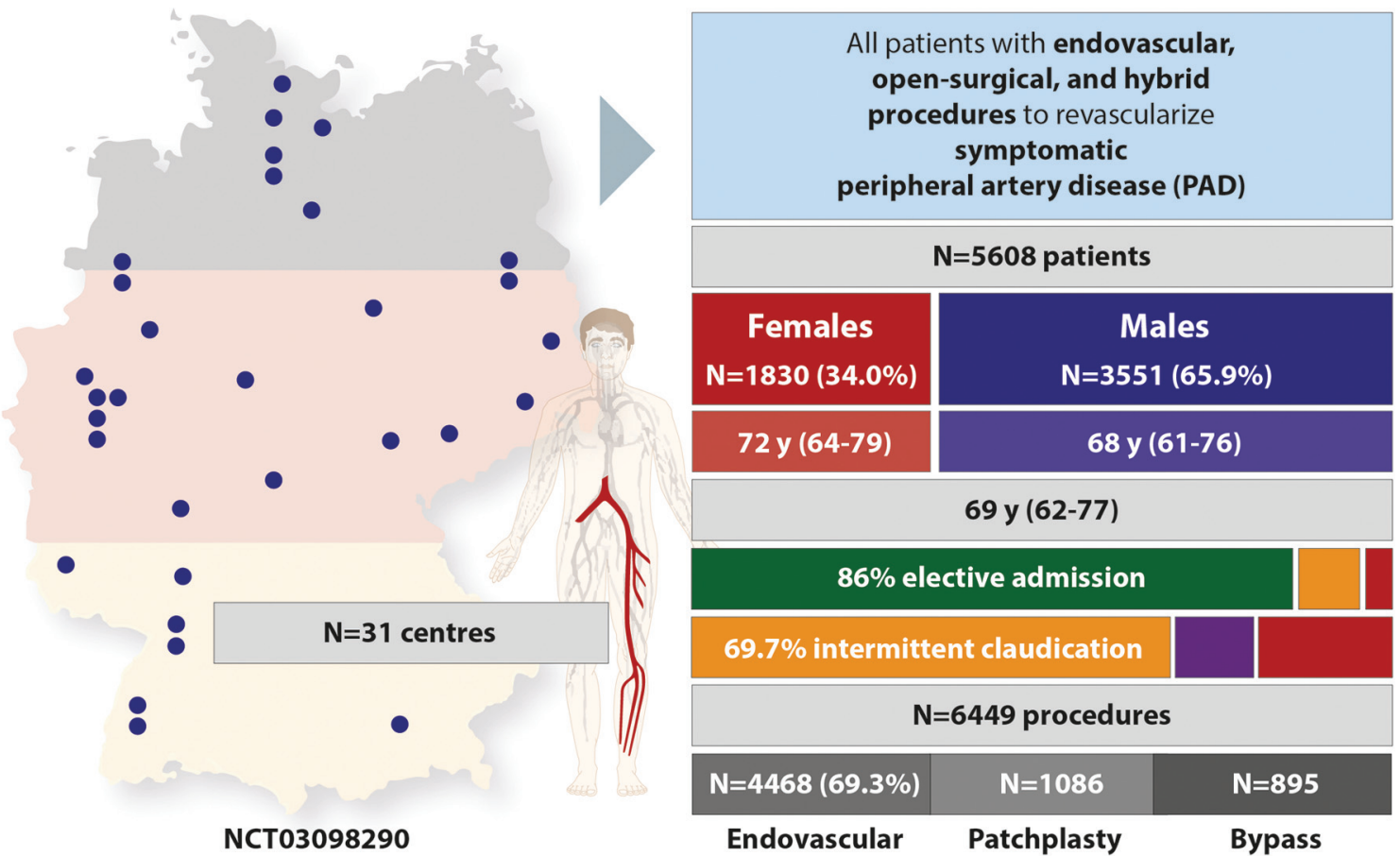

Figure 1. Central Illustration of this prospective multicentric cohort study of 5608 patients.

controlled trials (RCT) [6, 7], it appears reasonable to match conclusions derived from RCTs with high quality registry data better reflecting everyday clinical practice. For instance, in an ongoing debate concerning outcomes after drug-coated device treatment, RCTs reported excess long-term mortality while real-world data showed opposite results, emphasizing their complementary value $[8,9]$. While health insurance claims data offer particularly large samples for such purpose, clinical registries additionally offer the inclusion of more detailed parameters such as body mass index, lesion characteristics, blood pressure, and ankle-brachial-index.

The GermanVasc study included patients with symptomatic PAD who underwent either endovascular, opensurgical, or hybrid revascularisations between $1^{\text {st }}$ May 2018 and $31^{\text {st }}$ December 2020 at 31 German centres (Figure 1). The rationale and methods were published and registered a priori (clinicaltrials.gov NCT03098290) $[10,11]$. All indicators of outcome quality and additional variables collected in the current study were aligned by international Delphi consensus methods $[12,13,14]$. The data collection underwent both an independent randomsample validation and automated quality assurance.

The primary goal of the study was to quantify to which extent real-world treatment follow guideline recommendations.

The current report aims to present the baseline characteristics of the included patients.

\section{Material and methods}

This was a prospective longitudinal multicentre cohort study. The rationale and methods of the GermanVasc registry study were published a priori $[10,11]$ and additionally registered at Clinicaltrials.gov (NCT03098290) and the German Registry of Clinical Trials (DRKS00014649). A total of 18 ethical committees in affected German federal states confirmed the initial approval by the leading ethical committee at the medical association in Hamburg, Germany (PV5691). The European Union (EU) General Data Protection Regulation (GDPR) compliant GermanVasc registry platform was developed to follow the principles of privacy by design while collecting the personal and medical data relevant for the current study $[11,15,16]$. Results were reported using the STrengthening the Reporting of OBservational studies in Epidemiology (STROBE) statement [17].

\section{Inclusion and exclusion criteria}

All patients above 18 years who underwent either endovascular, open-surgical, or hybrid revascularisation for chronic symptomatic PAD between $1^{\text {st }}$ May 2018 and $31^{\text {st }}$ December 2020 at participating study centres were included if an explicit informed consent was available by the data subject. Patients with embolic acute limb ischaemia without history of chronic PAD were excluded. According to the modified Rutherford classification, patients selected for invasive revascularisation with mild, moderate, and severe claudication were pooled as intermittent claudication (IC). Patients selected with ischaemic rest pain, ulcer or necrosis, and non-healing amputation were pooled as chronic limb-threatening ischaemia (CLTI).

\section{Study variables}

The data collection in the current study followed three previous Delphi consensus studies on registry core elements and quality indicators for peripheral arterial revascularisation. These study variables were published 
elsewhere $[12,13,14]$. Variables were collected at baseline, after three, six, and twelve months of follow-up. In the current report, we present baseline characteristics as follow-up data collection is still ongoing.

In short, the following medical variables were collected during the baseline treatment: Age (years), sex (male, female, transgender), admission month and weekday, discharge month and weekday, urgency of the admission, living status, functional status, ambulation, discharge destination, weight (kilogram), height (meter), body mass index (BMI, calculated as $\mathrm{kg} / \mathrm{m}^{2}$ ), American Society of Anaesthesiologists (ASA) class, diabetes mellitus, glycohaemoglobin, renal insufficiency, most recent serum creatinine, current dialysis dependency, tobacco use (active, former), years since last smoking, current ischaemic heart disease, congestive heart failure, ejection fraction, cardiac arrhythmia, history of atrial fibrillation, chronic obstructive pulmonary disease (COPD), hypertension, prior peripheral arterial disease revascularisation, prior lower leg amputations, antiplatelets at the time of admission and at discharge, statins at the time of admission and at discharge, PCSK9-inhibitor at the time of admission and at discharge, vitamin $\mathrm{K}$ antagonist at the time of admission and at discharge, new/direct oral anticoagulants at the time of admission and at discharge, modified Rutherford classification (per side), foot infection (per side), ankle-brachial-index (ABI, per side, categorised), tissue loss (per side), postoperative myocardial infarction, postoperative stroke, postoperative new dialysis dependency, postoperative ankle-brachial-index (per side, categorized), postoperative unplanned amputation (per side), postoperative occlusion of index revascularisation, postoperative distal embolization, postoperative dissection, postoperative failure of graft or device, postoperative bleeding including pseudoaneurysm, postoperative compartment syndrome, postoperative wound infection, quality of life (WIQ and SF36).

\section{Statistical analysis}

Normality of data was tested using the Shapiro-Wilk-Test. We summarized the baseline characteristics of the patients with median and interquartile range (IQR) for nonnormally distributed variables with mean and standard deviation for normally distributed variables, and with percentages and Wald 95\% confidence interval (CI) for categorical variables. Missing values were handled by case exclusion for each analysis.

All statistical analyses were performed with SPSS version 25 (IBM Corporation, New York, USA). Visualization was performed with Adobe Illustrator version 24.1.2 (Adobe, San Jose, CA, USA).

\section{Results}

In total, 5608 patients $(34 \%$ females, median 69 years, IQR: 62-77) treated in 31 centres with 6449 procedures in total were registered from $1^{\text {st }}$ May 2018 through $31^{\text {st }}$
December 2020. External on-site visits were performed at least once in $100 \%$ of all study centres. Core characteristics were complete in $100 \%$ of all cases.

The baseline characteristics of the entire cohort and by occurrence of CLTI are presented in Table I. An urgent or emergent presentation at the study centre was documented in $12.5 \%$ (95\% CI: 11.6-13.4). Among a total of 6449 documented procedures provided to the study cohort, 4468 (69.3\%, 95\% CI: 68.1-70.4) were endovascular procedures.

Among all patients, 4.9\% (95\% CI: 4.4-5.5) were referred from another hospital or department to the study centre, and $0.8 \%$ (95\% CI: 0.6-1.1) were admitted from a nursery or rehabilitation facility. More than half of the cohort (54.9\%, 95\% CI: 53.5-56.2) exhibited at least one previous revascularisation of the lower extremities, and 5.5\% (95\% CI: 4.9-6.2) exhibited a previous major lower limb amputation before the index treatment.

In total, $4.6 \%$ (95\% CI: 4.1-5.2) of the patients needed assisted care or bedridden, while $18.0 \%$ (95\% CI: 17.0 19.0) were supplied with either prosthesis, assistive device, or wheelchair.

Regarding the overall physical status according to the ASA classification, $65.1 \%$ were either classified as a patient with mild systemic disease (30.6\%, 95\% CI: 29.4-31.8) or severe systemic disease (34.5\%, 95\% CI: 33.2-35.7). Among all patients, 3.1\% (95\% CI: 2.7-3.6) exhibited a severe systemic disease that is a constant threat to life.

\section{Body mass index, hypertension, smoking, and diabetes}

The median body mass index (BMI) of the entire cohort was $26.0 \mathrm{~kg} / \mathrm{m}^{2}$ (95\% CI: $23.4-29.3$ ), and 20\% (95\% CI: 19.0-21.1) were obese according to the threshold of $30 \mathrm{~kg} / \mathrm{m}^{2}$.

82.2\% (95\% CI: 81.1-83.2) had a history of hypertension. $44.3 \%$ (95\% CI: 43.0-45.6) of the entire cohort reported current smoking at the time being selected for invasive revascularisation, and 30.7\% (95\% CI: 29.5-31.9) were former smoker with a median quit time of 15 years (IQR: 6-26).

35.7\% (95\% CI: 34.5-37.0) were diagnosed with diabetes. Among these patients, the median HbA1C value was 7 (IQR: 6-8).

\section{Cardiac risk}

One third of the cohort was diagnosed with ischemic heart disease. Among all patients, 29.5\% (95\% CI: 28.3-30.8) were asymptomatic at the time of presentation, $5.3 \%$ (95\% CI: 4.7-5.9) exhibited angina only during physical activity, and $1.3 \%$ (95\% CI: 1.1-1.7) exhibited symptoms at everyday living activities or at rest.

14.9\% (95\% CI: 14.0-15.9) of the patients reported any history of coronary artery revascularisation, and $16.8 \%$ (95\% CI: $15.8-17.8)$ had a history of myocardial infarction. 
Table I. Baseline characteristics of this cohort including 5608 patients with invasive revascularisation for symptomatic peripheral artery disease. If not otherwise indicated, all values are presented as percentage (\%) with 95\% confidence interval

\begin{tabular}{|c|c|c|c|c|c|c|}
\hline \multirow{3}{*}{$\begin{array}{l}\text { Number of patients } \\
\text { Patient age, years (median, interquartile range) }\end{array}$} & \multirow{2}{*}{\multicolumn{2}{|c|}{$\begin{array}{c}\begin{array}{c}\text { Total } \\
\text { cohort }\end{array} \\
5608\end{array}$}} & \multirow{2}{*}{\multicolumn{2}{|c|}{$\begin{array}{c}\begin{array}{c}\text { Chronic limb- } \\
\text { threatening } \\
\text { ischaemia }\end{array} \\
1676(29.9 \%)\end{array}$}} & \multirow{2}{*}{\multicolumn{2}{|c|}{$\begin{array}{c}\begin{array}{c}\text { No chronic limb- } \\
\text { threatening } \\
\text { ischaemia }\end{array} \\
3932(70.1 \%)\end{array}$}} \\
\hline & & & & & & \\
\hline & 69 & $62-77$ & 72 & $64-80$ & 68 & $61-76$ \\
\hline Octogenarians & 15.7 & $14.8-16.7$ & 25.4 & $25.4-27.6$ & 11.6 & $10.6-12.6$ \\
\hline Females & 34.0 & $32.7-35.3$ & 34.3 & $32.0-36.7$ & 33.8 & $32.3-35.4$ \\
\hline Urgent or emergent presentation & 12.5 & $11.6-13.4$ & 31.7 & $29.5-34.0$ & 4.2 & $3.6-4.9$ \\
\hline Referred from another hospital & 4.9 & $4.4-5.5$ & 11.2 & $9.8-12.8$ & 2.2 & $1.8-2.7$ \\
\hline Admitted from nursery/rehab & 0.8 & $0.6-1.1$ & 2.3 & $1.7-3.2$ & 0.3 & $0.1-0.5$ \\
\hline Needed assisted care/bedridden & 4.6 & $4.1-5.2$ & 11.5 & $10.0-13.1$ & 1.6 & $1.3-2.1$ \\
\hline Ambulation with any assistive device or bedridden & 18.0 & $17.0-19.0$ & 39.4 & $37.0-41.8$ & 8.7 & $7.9-9.7$ \\
\hline ASA Class III & 34.5 & $33.2-35.7$ & 39.4 & $37.0-41.8$ & 29.8 & $28.4-31.3$ \\
\hline ASA Class IV & 3.1 & $2.7-3.6$ & 6.6 & $5.4-7.9$ & 1.6 & $1.2-2.1$ \\
\hline Body mass index (median, interquartile range) & 26.0 & $23.4-29.3$ & 25.8 & $22.9-29.3$ & 26.1 & $23.7-29.3$ \\
\hline Body mass index $>30 \mathrm{~kg} / \mathrm{m}^{2}$ & 20.0 & $19.0-21.1$ & 21.0 & $19.1-23.0$ & 19.6 & $18.4-20.9$ \\
\hline Diabetes mellitus & 35.7 & $34.5-37.0$ & 47.4 & $45.0-49.8$ & 30.7 & $29.2-32.1$ \\
\hline Chronic renal failure & 22.1 & $21.0-23.2$ & 32.5 & $30.2-34.8$ & 17.7 & $16.5-18.9$ \\
\hline Dialysis dependency & 2.5 & $2.1-2.9$ & 5.4 & $4.4-6.6$ & 1.3 & $0.9-1.6$ \\
\hline Current smoker & 44.3 & $43.0-45.6$ & 37.7 & $35.3-40.0$ & 46.3 & $44.8-47.9$ \\
\hline Former smoker & 30.7 & $29.5-31.9$ & 30.5 & $28.3-32.8$ & 30.2 & $28.8-31.7$ \\
\hline Quit time, years (median, interquartile range) & 15 & $6-26$ & 16 & $7-30$ & 13 & $5-24$ \\
\hline Asymptomatic ischaemic heart disease & 29.5 & $28.3-30.8$ & 34.2 & $31.9-36.5$ & 27.5 & $26.1-28.9$ \\
\hline Angina symptoms during physical activity & 5.3 & $4.7-5.9$ & 6.6 & $5.5-7.9$ & 4.7 & $4.0-5.4$ \\
\hline Angina symptoms at everyday living activities or at rest & 1.3 & $1.1-1.7$ & 1.7 & $1.2-2.5$ & 1.2 & $0.9-1.6$ \\
\hline History of coronary artery revascularisation & 14.9 & $14.0-15.9$ & 18.1 & $16.3-20.0$ & 13.6 & $12.5-14.7$ \\
\hline History of myocardial infarction & 16.8 & $15.8-17.8$ & 20.4 & $18.4-22.4$ & 15.3 & $14.2-16.4$ \\
\hline History of congestive heart failure & 17.0 & $16.0-18.0$ & 23.5 & $21.5-25.5$ & 13.9 & $12.8-15.0$ \\
\hline Ejection fraction, \% & 50 & $40-55$ & 45 & $35-55$ & 51 & $42-55$ \\
\hline History of cardiac arrhythmias & 18.7 & $17.7-19.8$ & 23.5 & $21.5-25.6$ & 14.9 & $13.8-16.0$ \\
\hline History of chronic obstructive pulmonary disease & 11.6 & $10.7-12.4$ & 12.7 & $11.2-14.4$ & 10.9 & $9.9-11.9$ \\
\hline History of hypertension & 82.2 & $81.1-83.2$ & 74.8 & $73.4-76.2$ & 81.9 & $79.9-83.7$ \\
\hline History of any lower extremity revascularisation & 54.9 & $53.5-56.2$ & 56.1 & $53.7-58.5$ & 49.3 & $47.8-50.9$ \\
\hline History of any lower extremity amputation & 5.5 & $4.9-6.2$ & 13.4 & $11.8-15.1$ & 2.1 & $1.7-2.6$ \\
\hline
\end{tabular}

ASA: American Society of Anaesthesiologists.

A history of congestive heart failure was reported in 17.0\% (95\% CI: 16.0-18.0), while a median left ventricular ejection fraction of 50\% (IQR: 40-55) was documented in these patients. $18.7 \%$ (95\% CI: 17.7-19.8) reported any history of clinically relevant cardiac arrhythmias.

\section{Other risk factors}

A chronic renal failure was apparent in $22.1 \%$ (95\% CI: 21.0-23.2) of the cohort, while 2.5\% (95\% CI: 2.1-2.9) were dependent from chronic dialysis.

A chronic obstructive pulmonary disease was reported by $11.6 \%$ (95\% CI: $10.7-12.4)$ of the patients.

\section{Invasive procedures}

A total of 6449 invasive procedures were registered. Among all registered revascularisations, 69.3\% (95\% CI: 68.1-70.4) were endovascular procedures $(n=4468)$. A total of 1285 drug-coated balloons and 292 drug-eluting stents were registered. Vascular surgeons were actively involved in the procedure in $43.0 \%$ (95\% CI: $41.5-44.4)$, interventional radiologists in 33.5\% (95\% CI: 32.1-34.9), and interventional internists in $34.4 \%$ (95\% CI: $33.1-35.9$ ) of the procedures. A total of $17.8 \%$ (95\% CI: $16.7-19.0$ ) of the procedures involved at least two medical specialties. $8.7 \%$ (95\% CI: 7.9-9.6) of the procedures were performed as hybrid approach with cut down. In 52.6\% (95\% CI: 51.1-54.1) of the registered procedures, a total of 2351 closure devices were documented.

\section{Antiplatelets and statins}

At the time of admission, 84.7\% (95\% CI: 83.7-85.6) of the patients were on antiplatelet medication, while $67.5 \%$ (95\% CI: 66.2-68.7) were taking statins. After discharge, the prescription rate of antiplatelets was $93.5 \%$ (95\% CI: 92.8-94.1) and 76.5\% (95\% CI: 75.4-77.6) for statins. 


\section{Risk profile by occurrence of chronic limb-threatening ischaemia}

Among the entire cohort, 1676 (29.9\%, 95\% CI: 28.7-31.1) patients presented with either ischaemic rest pain or ischaemic wound healing disorders. In CLTI patients, the proportion of octogenarians, urgent or emergent presentation, referral from another hospital, and referral from nursery or rehabilitation facilities was higher when compared with patients without CLTI symptoms (Table I). The overall risk profile was more pronounced in CLTI patients concerning higher ASA class, diabetes, chronic renal failure, cardiac disease, and previous lower extremity revascularisation or amputation.

\section{Discussion}

This large prospective observational cohort study evaluated the everyday clinical practice at 31 vascular centres in Germany. More than 5600 patients were treated by approximately 6500 endovascular and open-surgical procedures for chronic symptomatic PAD. The protocol of the current study was published a priori, and the study data underwent a rigorous validation by an external randomsample and risk-based quality assurance.

The included patients, especially those suffering from CLTI, exhibited a severe multimorbidity and multiple cardiovascular risk factors. One third of the cohort were patients with severe systemic disease, and more than three percent were in a life-threatening condition.

There is growing evidence for a wide variation of practice patterns between countries, centres, and registries. The reasons, however, are mostly unknown. In the United Kingdom, the Getting It Right First Time (GIRFT) programme identified such variations as possible room for improvement [18]. The 2018 GIRFT report found that many patients needing urgent surgery face long or uncertain waits, and a "lack of consistency in the approach taken to the same condition - with different providers choosing different surgical methods in apparently similar circumstances" [19]. Globally, a recent comparison of population-based registries in eleven countries revealed that patient selection and treatment modality varied widely for the proportion of patients with intermittent claudication (6\% in Italy and 69\% in Russia) and endovascular techniques (24\% in Russia and $88 \%$ in Italy) [2]. Confirming these previous studies, one third of the current study cohort exhibited symptoms of a CLTI and 70\% among all procedures included endovascular techniques.

Interestingly, in the current study, more than half of the cohort had a history of any lower extremity revascularisation, confirming previous reports using longitudinal data $[18,20]$. This finding further emphasizes the importance to use patient-linked instead of rather procedure-related data since these redundant treatments would otherwise distort results derived from unlinked databases. However, to date, longitudinally linked multicentre registry data from
Germany remains scarce. The RECCORD registry of the society for angiology enrolled 1000 patients with endovascular treatment of symptomatic PAD (25\% with CLTI, 35\% females, mean age 70 years) but the data collection primarily covered treatments performed by a single medical specialty [21]. In the current study, three different medical specialties were almost equally involved in the endovascular procedures, emphasizing the importance to include all specialties in data collections on endovascular treatments. Against that backdrop it appears noteworthy to highlight that all valid practice guidelines recommend a multidisciplinary approach but there is evidence suggesting an insufficient adherence concerning multidisciplinary team decisions in PAD treatment [22]. From 2013 through 2014, the German national registry for first line treatment strategies in patients with critical limb ischemia (CRITISCH register) collected multicentre data on 1200 patients suffering from CLTI from 27 selected centres. However, considering the current treatment reality and increasingly frequent invasive treatment of patients with intermittent claudication, it appears likewise important to cover the full spectrum of disease stages in realworld data [23].

Considering the ongoing COVID-19 pandemic, the unfavourable risk profile of the current study cohort deserves thoroughly reflection. More and more studies report a growing number of predictors for a severe illness. Males, octogenarians, patients with cardiovascular disease, diabetes, chronic liver and renal disease, chronic pulmonary disease, malignancy, and those with obesity and smoking are at higher risk when compared to the healthy population [24]. Notably, the current study included a highly vulnerable cohort affected by almost all of these severe comorbidities. It may be reasonable to focus on tertiary prevention and re-evaluate vaccination strategies to protect this central target population.

Against that backdrop, the fact that nearly half of the study cohort reported active smoking at the time being selected for invasive revascularisation appears striking. The central importance of smoking cessation was not only highlighted in all valid practice guidelines but is commonly accepted to be one of the most important drivers of early mortality in numerous global cohorts $[3,4,5,25,26]$. Together with the high proportion of obese patients in the current study, the modifiable risk factors may need more attention by the vascular community.

\section{Limitations}

Besides many strengths, there are also limitations. First, the patient selection and choice of treatment approach was left to the discretion of the physicians. This prospective observational study collected routinely collected data, and the non-random assignment makes it impossible to derive a causal relationship between treatment strategy and outcomes. Second, the study comprises 31 high-volume centres of about 650 hospitals providing vascular health benefits to the target population in Germany. Therefore, 
a selection bias cannot be ruled out although centres with a large variety of characteristics from all over Germany were involved.

The GermanVasc data collection will be used for comprehensive outcome research during the following years. With a follow-up of one year and beyond, a meaningful comparison of commonly accepted quality indicators and objective performance goals is planned. The peculiar risk profile of this vulnerable cohort and its interaction with the intervention-outcome relationship will be further evaluated. The impact of renal insufficiency, cardiac comorbidities, female sex, and diabetes is of special interest. In the beginning of 2021, a medical device module was implemented to the registry platform in order to collect unique device identifiers (UDI) for the evaluation of long-term outcomes (www.mdepinet.de).

\section{Conclusions}

This study reports baseline characteristics from a large validated all-comer prospective cohort in Germany. The cohort covers a broader range of disease severity and types of interventions than usually found in trials. In future studies, the relation between treatment at baseline and outcomes will be analysed in more detail.

\section{References}

1. Fowkes FG, Rudan D, Rudan I, Aboyans V, Denenberg JO, McDermott MM, et al. Comparison of global estimates of prevalence and risk factors for peripheral artery disease in 2000 and 2010: A systematic review and analysis. Lancet. 2013;382:1329-40.

2. Behrendt CA, Sigvant B, Kuchenbecker J, Grima MJ, Schermerhorn M, Thomson I, et al. International variations and gender disparities in the treatment of peripheral arterial occlusive disease - A report from VASCUNET and the International Consortium of Vascular Registries. Eur J Vasc Endovasc Surg. 2020;60:873-80.

3. Aboyans V, Ricco JB, Bartelink MEL, Bjorck M, Brodmann M, Cohnert T, et al. Editor's Choice - 2017 ESC guidelines on the diagnosis and treatment of peripheral arterial diseases, in collaboration with the European Society for Vascular Surgery (ESVS). Eur J Vasc Endovasc Surg. 2018;55:305-68.

4. Gerhard-Herman MD, Gornik HL, Barrett C, Barshes NR, Corriere MA, Drachman DE, et al. 2016 AHA/ACC guideline on the management of patients with lower extremity peripheral artery disease: A report of the American College of Cardiology/ American Heart Association Task Force on clinical practice guidelines. Circulation. 2017;135:e726-79.

5. Frank U, Nikol S, Belch J, Boc V, Brodmann M, Carpentier PH, et al. ESVM Guideline on peripheral arterial disease. VASA. 2019;48:1-79.

6. Menard MT, Farber A. The BEST-CLI trial: A multidisciplinary effort to assess whether surgical or endovascular therapy is better for patients with critical limb ischemia. Sem Vasc Surg. 2014;27:82-4.

7. Hunt BD, Popplewell MA, Davies H, Meecham L, Jarrett H, Bate $\mathrm{G}$, et al. BAlloon versus Stenting in severe Ischaemia of the Leg-3 (BASIL-3): Study protocol for a randomised controlled trial. Trials. 2017;18:224

8. Behrendt CA, Sedrakyan A, Peters F, Kreutzburg T, Schermerhorn M, Bertges DJ, et al. Editor's Choice - Long term survival after femoropopliteal artery revascularisation with paclitaxel coated devices: A propensity score matched cohort analysis. Eur J Vasc Endovasc Surg. 2020;59:587-96.

9. Behrendt CA, Peters F, Mani K. The swinging pendulum of evidence: Is there a reality behind results from randomised trials and real world data? Lessons learned from the paclitaxel debate. Eur J Vasc Endovasc Surg. 2020;59:510-1.

10. Behrendt CA, Riess HC, Schwaneberg T, Heidemann F, Tsilimparis N, Larena-Avellaneda AA, et al. Complex endovascular treatment of intact aortic aneurysms: An analysis of health insurance claims data. Gefasschirurgie. 2018;23:32-8.

11. Behrendt CA, Schwaneberg T, Hischke S, Muller T, Petersen T, Marschall U, et al. Data privacy compliant validation of health insurance claims data: The IDOMENEO approach. Gesundheitswesen. 2020;82:S94-100.

12. Behrendt CA, Bjorck M, Schwaneberg T, Debus ES, Cronenwett J, Sigvant B, et al. Editor's Choice - Recommendations for registry data collection for revascularisations of acute limb ischaemia: A Delphi consensus from the international consortium of vascular registries. Eur J Vasc Endovasc Surg. 2019;57:816-21.

13. Behrendt CA, Bertges D, Eldrup N, Beck AW, Mani K, Venermo $\mathrm{M}$, et al. International consortium of vascular registries consensus recommendations for peripheral revascularisation registry data collection. Eur J Vasc Endovasc Surg. 2018;56:217-37.

14. Riess HC, Debus ES, Schwaneberg T, Hischke S, Maier J, Bublitz $\mathrm{M}$, et al. Indicators of outcome quality in peripheral arterial disease revascularisations - a Delphi expert consensus. VASA. 2018;47:491-7.

15. Petersen T, Blochberger M, Mueller T, Federrath H. [Sichere und datenschutzgerechte Umsetzung medizinischer Register]. DuD. 2019;43:507-12.

16. Bavendiek K, Mueller T, Wittner F, Schwaneberg T, Behrendt CA, Schulz W, et al. Automatically Proving Purpose Limitation in Software Architectures. In: Dhillon G, Karlsson F, Hedström K, Zúquete A (eds.). ICT Systems Security and Privacy Protection. SEC 2019. IFIP Advances in Information and Communication Technology. Vol. 562. Cham: Springer; 2019. 345-58.

17. von Elm E, Altman DG, Egger M, Pocock SJ, Gotzsche PC, Vandenbroucke JP, et al. The Strengthening the Reporting of Observational Studies in Epidemiology (STROBE) statement: Guidelines for reporting observational studies. J Clin Epidemiol. 2008;61:344-9.

18. Gray WK, Day J, Horrocks M. Outcomes for angioplasty and bypass lower limb revascularisation procedures for limb salvage in England: Findings from the getting it right first time programme. Eur J Vasc Endovasc Surg. 2020;60:711-9.

19. Behrendt CA, Lyons O. Is England getting it right yet? Potential lessons for Europe. Eur J Vasc Endovasc Surg. 2020;60:720.

20. Kreutzburg T, Peters F, Riess HC, Hischke S, Marschall U, Kriston L, et al. Editor's Choice - Comorbidity patterns among patients with peripheral arterial occlusive disease in Germany: A trend analysis of health insurance claims data. Eur $J$ Vasc Endovasc Surg. 2020;59:59-66.

21. Malyar N, Stausberg J, Langhoff R, Tatò F, Kalka C, Ito WD, et al. Demographic and procedural characteristics in the RECording COurses of vasculaR Diseases (RECCORD) registry - the first 1000 patients. VASA. 2020;49:382-8.

22. Behrendt CA, Kolbel T, Schwaneberg T, Diener H, Hohnhold R, Debus ES, et al. Multidisciplinary team decision is rare and decreasing in percutaneous vascular interventions despite positive impact on in-hospital outcomes. VASA. 2019;48:262-9.

23. Bisdas T, Borowski M, Torsello G. First-Line Treatments in Patients With Critical Limb Ischemia Collaborators. Current practice of first-line treatment strategies in patients with critical limb ischemia. J Vasc Surg. 2015;62:965-73.e3.

24. Docherty AB, Harrison EM, Green CA, Hardwick HE, Pius R, Norman L, et al. Features of 20133 UK patients in hospital with COVID-19 using the ISARIC WHO Clinical Characterisation Protocol: Prospective observational cohort study. BMJ. 2020;369: m1985 
25. Janssen F, El Gewily S, Bardoutsos A. Smoking epidemic in Europe in the 21st century. Tob Control. 2020. Online ahead of print.

26. Rentería E, Jha P, Forman D, Soerjomataram I. The impact of cigarette smoking on life expectancy between 1980 and 2010: A global perspective. Tob Control. 2016;25:551-7.

\section{History}

Submitted: 07.05.2020

Accepted after revision: 17.06.2021

Published online: 19.07.2021

\section{Acknowledgement}

The authors are grateful for the data submission and valuable contributions to the manuscript by Erwin Blessing (SRH-ClinicKarlsbad-Langensteinbach, Karlsbad, Germany), Matthias Heinrich Tenholt (Theresien Hospital Mannheim, Mannheim, Germany), Jörg Teßarek (St. Boniface Hospital Lingen, Lingen, Germany), Martin Czerny (University Heart Centre Freiburg, Freiburg, Germany), Sebastian Schellong (Municipal Hospital Dresden Friedrichstadt, Dresden, Germany), Arndt Hribaschek (Magdeburg Hospital, Magdeburg, Germany), Irene Hinterseher (Charité Universitätsmedizin Berlin, corporate member of Freie Universität Berlin, Humboldt-Universität zu Berlin, and Berlin Institute of Health, Campus Benjamin Franklin), Amin Moussa (Agaplesion Diakonie Clinics Kassel, Kassel, Germany), Dimitrios Theodosiou (Agaplesion Diakonie Clinics Hamburg, Hamburg, Germany), Martin Storck (Karlsruhe Municipal Clinic, Karlsruhe, Germany), Alexander Stehr (Evangelical Hospital Mühlheim an der Ruhr, Mühlheim a.d.R., Germany), Sven Seifert (Chemnitz Hospital gGmbH, Chemnitz, Germany), Roland Stöbe (Carl-Thiem-Clinic Cottbus, Cottbus, Germany), Artis Knapsis (University Hospital Düsseldorf, Düsseldorf, Germany), Markus Siggelkow (Imland Clinic Rendsburg, Rendsburg, Germany), Markus Kleemann (University Hospital Schleswig-Holstein, Lübeck Campus, Lübeck, Germany), Gerd Lulay (Rheine Clinic I Mathias Spital, Rheine, Germany), Richard Kellersmann (Fulda Clinic, Fulda, Germany), Axel Larena-Avellaneda (Asklepios Clinic Altona, Hamburg, Germany), Joerg Herold and Rupert Bauersachs (both Clinical Center Darmstadt, Darmstadt, Germany), Thomas Knöfel (Central Clinic Bad Berka, Bad Berka, Germany), Klaus Rilling (University
Hospital Schleswig-Holstein, Campus Kiel, Kiel, German), and Henrik Christian Rieß (University Heart and Vascular Centre UKE Hamburg, Germany).

The authors would like to thank Professor Hannes Federrath, Tobias Müller, Tom Petersen, Joshua Stock, and Pascal Wichmann from the research group "Security in Distributed Systems" at the Informatics Department, University of Hamburg, Germany. The authors would also like to thank Thea Kreutzburg, Nicol Rona, Breda Geertz, and Jenny Kuchenbecker from the research group GermanVasc at the University Medical Center Hamburg-Eppendorf, Germany. Numerous physicians, researchers, study nurses, and administrative staff at the participating study centres helped to collect and validate the study data. The authors are grateful for their great commitment.

\section{Conflict of interest}

The authors declare that there are no conflicts of interest.

\section{Authorship}

Artur Kotov and Frederik Peters shared authorship.

\section{Funding}

The IDOMENEO and RABATT studies were funded by the Innovations Fund of the German Federal Joint Committee (grant number 01VSF16008, grant number 01VSF18085, PI: CAB).

\section{ORCID}

Christian-Alexander Behrendt

(D) https://orcid.org/0000-0003-0406-3319

\section{Correspondence address}

Assoc. Prof. Dr. Christian-Alexander Behrendt

Research Group GermanVasc

University Medical Center Hamburg-Eppendorf

Martinistraße 52

20246 Hamburg

Germany

behrendt@hamburg.de 